\title{
Synthesis, structure, and properties of new phthalocyanines
}

\author{
Nagao Kobayashi ${ }^{* a}$ and Clifford C. Leznoff ${ }^{\star b}$ \\ a Department of Chemistry, Graduate School of Science, Tohoku University, Sendai 980-8578, Japan \\ ' Department of Chemistry, York University, Toronto, Ontario M3J IP3, Canada
}

\begin{abstract}
Many low symmetry and/or deformed phthalocyanines (Pcs) have been synthesized, separated, and their spectroscopic and electrochemical properties elucidated in the last decade, mainly because of improvement in separation techniques of reaction mixtures and development of instruments and computers. In this overview, some advances in the chemistry of Pcs are surveyed. In recent years, many types of alkynyl Pcs, various isomers of low symmetry Pcs, severely sterically deformed Pcs, and chiral Pcs have been prepared and some of their properties are reproduced by quantum mechanical calculations, which have not been achieved previously. Also, in the field of Pc sandwich complexes, properties of hetero double- and triple-decker complexes can be compared, since many types of compounds are now available. Some hetrodinuclear planar dimers have also been reported in the last decade. Copyright 12004 Society of Porphyrins \& Phthalocyanines.
\end{abstract}

KEYWORDS: phthalocyanine, symmetry, deformation, spectroscopy, electrochemistry.

The first phthalocyanine $(\mathrm{Pc})$ was synthesized accidentally in 1907 when o-cyanobenzamide was heated at high temperature [1]. In 1934, the structure of this metal-free Pc was elucidated by Linstead [24] so that the year 2004 is the 70th year after the confirmation of the structure of Pcs. Since then, monomeric Pcs have been used in many practical fields, and they are now called "the compounds of the 21 st century" with this expectation, since they have a great diversity of possibilities [5].

The chemistry of low symmetry Pcs were elucidated some time ago. For example, tribenzo tetraazaporphyrin was reported as early as 1955 by Elvidge and Linstead [6], and tetrabenzotriazaporphyrin was synthesized in the late 1930 s $[7,8]$. Although not many researchers were engaged in the chemistry of low symmetry Pcs [9-11], this field underwent a revival by many Pc chemists when Luk'yanets [12], Leznoff [13], and Kobayashi [14, 15] independently reported the synthesis and intriguing spectroscopic properties of several low symmetry Pc derivatives around 1990. Since then, many papers have reported on the synthesis and properties of Pc analogues [9. $11,16]$. Some representative examples are mentioned below. 1) Makarova et al. prepared opposite and adjacent diazatetrabenzporphyrin [12] and this is still the one and only paper which reports a clear separation of the two isomers. Although the former $D_{2 h}$ type derivatives showed a well-split $\mathrm{Q}$ band, the latter $C_{2 v}$ type species does not show a split $\mathrm{Q}$ band, and this has been explained theoretically [17]. 2) Leznoff and McKeown reported synthetic details leading to tetrabenzotriazaporphyrins [13] and these compounds showed electrochemical [18] and spectroscopic [19] properties closer to Pcs than to tetrabenzoporphyrins. 3) A ZnPc having $D_{2 h}$ symmetry was reported in 1992 [15], and it showed a four-peak $Q$ band different from the two-peak $Q$ band of normal metal Pcs with $D_{4 h}$ symmetry. 4) Pc analogues in which one benzene ring of the $\mathrm{Pc}$ is substituted with other aromatic rings or substituted benzenes are obtained by ring-expansion of what were first called subPcs in 1990 [14]; Since then more than a hundred papers have appeared on subPcs and low symmetry Pcs derived by this method $[20,21]$. The resulting $C_{2 v}$ type compounds generally show a split 
$Q$ band duc to lowering of the molecular symmetry, and the position of the $Q$ band can be finoly adjusted by the type and kind of introduced aromatic rings. 5) By comparing the electrochemical data and the nesults of molecular ortital calculations of a series of Pe compounds having difterent $\pi$ size, a solution was given to a long-tanding question as to why the HOMO destabilizes, white the LUMO remalns at essentially the same level upor ting expandion. That is, the coeffeient of carbons of the HOMO is substantlally large even on the fused benzene carbons while that of the LUMO is amall, In other words, the HOMO spreads outward whils the LUMO does not enlarge oven if aromatic ringa are fused to the periphery of the letranzaporphyrins $(221,6)$ The splitting and the shift of the $\mathrm{Q}$ band of Po-like compounds aynthesized from phithalonitrile and 2,3-naphthalonterile cen be explained by symmeiry-adapted perturbation theory, but the NMR date of these compounds cannot be explained by a simple model $[23,24]$. This ldea also holds when two types of phthalonituriles are condensed to form low aymmetry Pe derivatives (25). 7) The chemistry of trinzolephthalocyanines has been developed by Torros and coworkers [26], Decause of the lowering of moleetlar symmetry and the presence of the triazole ting they show pecullar absorption spoctra; the $Q$ band lles at shontor wavolength and weak but the Sorot band appears at longer wevolength than the corresponding Pcs. Furthermore. Tomes and coworkers reported $D_{24}$ type, expanded triazole compounds which can accommodate two transition metal fons in their cavity [27]. Since these type of compounds are $28 \pi$ systems, it might be interesting to pursue their spectroscopic properties. 8) New $\mathrm{Pc}$ derivatives that have metal coordination sites on the Pc periphery, one of which is called gemini porphyrazine [28] have been cesigned by the group of Hoffman and Barrett. By coordination of metals to these sites, the absorption spectri change markedly (generally broadens) tindleating that the perturbation by coordination of mo f 1 quite signifcant. 9) The group of Hoffman and Barrett also sucecedod in synthesizing what we cull see0-porphyrazine [29], where a pyrrole ring of totraazaporphyrin is opened. Although the $\pi$ system is one of the stmallest among the porphyrin family, thetr $O$ band shifis to ca 700 nn when amino groups wero linked to the opened ring. 10) Leznoff developed a novel routc leading to unsymmetrical Pcs, which is now called "the polymer support method" [30] This is a very clean system so long as a phithlonitrilo unit can be linked. to a polymer, since symmetrical Pcs produced in the reaction can be eluted out from the polymer resin. The chemistry of low symmetry Pes have thus been extensively developod, and it appeare more difficult to obtain new systeme. However, it should bo stressed that it is now possible to soparate all compounds shown in Fig. 1 by use of high quality TLC plares

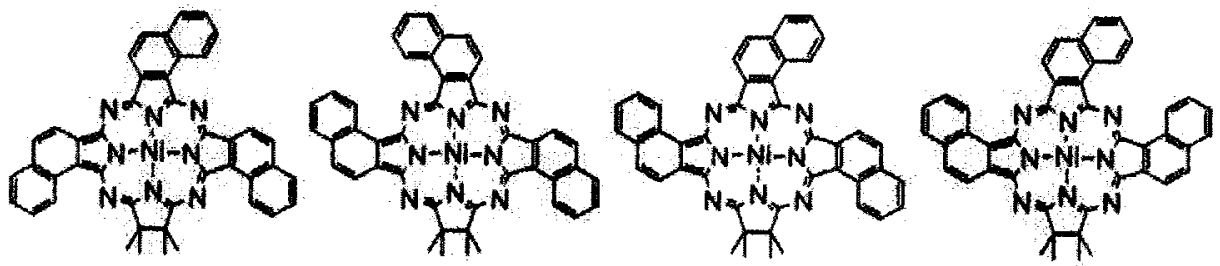

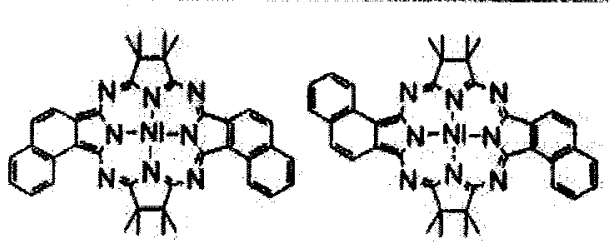

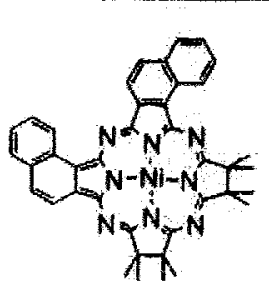

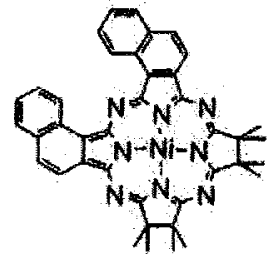

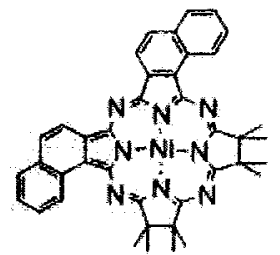

Fia. 1. Low symmeirical tetramzaporphyrins prepared from condensation between 2,3-dicyanonaphthalene and tetramethylsuccinonitrile 
and HPLC [31]. New low symmetry Pcs, such as structurally distorted Pcs, have been reported in the past few years. These are attained by linking eight large or long substituent groups at the benzene ring closest to the Pc core. Cook's group was the first who reported these kinds of Pcs [32]. In particular, when eight alkylthio groups were used, the Pc became red in color because of the emergence of a band at ca. $500 \mathrm{~nm}[33]$. In a similar manner, the use of phenyl groups also helps to severely distort the Pc plane [34]. When iron was inserted into its center and isocyanide groups were coordinated as axial ligands to form $D_{2 d} \mathrm{FePc}$, it showed a charge-transfer band beyond the $Q$ band, since the CT band which is forbidden in $D_{4 h}$ symmetry became allowed [35]. Optically active Pcs are also becoming popular [36] Representative examples include long alkyl chain- [37], small chiral ring- [38], helicene[39], and binaphthyl-substituted Pcs [40, 41]. In addition, vibrational $C D$ spectra of synthetic $\mathrm{Pc}$ derivatives were reported recently as the first example of VCD spectra for synthetic porphyrins and Pcs (Fig. 2) [42]. Chiral Pc enantiomers having rigid, non-aromatic small molecules give strong VCD signals. The introduction of linear triple bonds opened new fields in Pc chemistry. For example, Pcs containing eight triple bonds at the benzene ring furthest from the Pc core show red-shifted $Q$ bands $[43,44]$, and triple bonds helped in synthesizing dimers and oligomers of Pcs $[16,45]$ in which the mutual positions of the constituting monomer unit are fixed. Planar homodinuclear Pcs were reported as early as 1986 [46]. Since then, several types of heterodinuclear Pc dimers have been synthesized [47]. Representative examples include Torres's hemiporphrazine ring-fused $\mathrm{Pc}$ [48] and Kobayashi's pyrazinoporphyrazine-fused Pc [49]. Both of these compounds show spectra different from the superimposition of the spectra of constituting monomers. In the field of heterodinuclear lanthanide sandwich Pc complexes, Jiang's group contributed considerably in the past several years. To date, they have succeeded in preparing heterodimers consisting of octaethylporphyrin (OEP), tetraphenylporphyrin (TPP) and its derivatives, $\mathrm{Pc}$ and naphthalocyanine $(\mathrm{Nc})$ $[50,51]$. It is particularly important to prepare a series of compounds to prevent mistakes, for example, in assigning
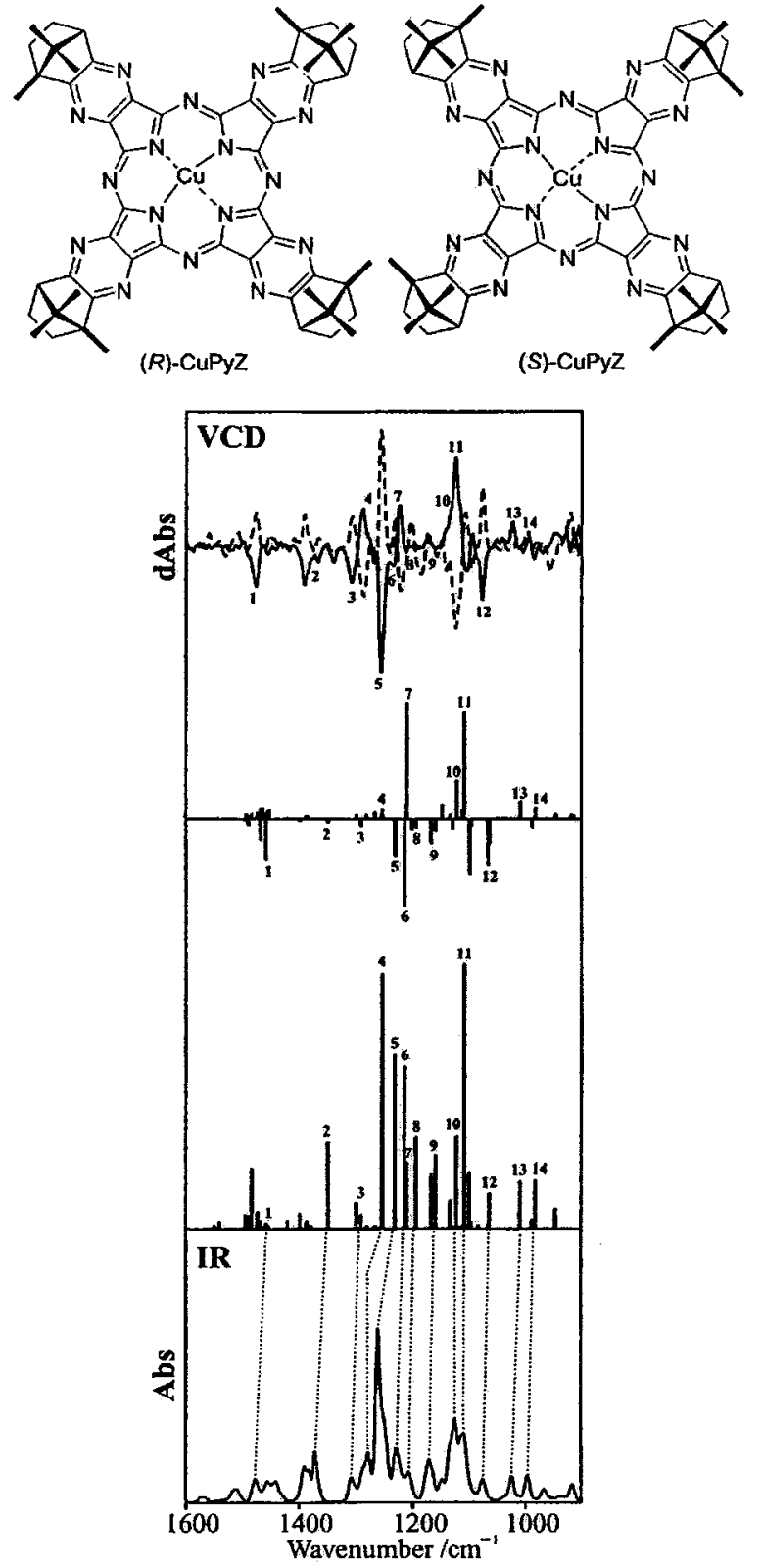

Fig. 2. Structures of $(R)-(-)$ - and $(S)$-(t)-camphor-substituted tetrapyrazino-porphyrazines and their experimental IR (bottom) and vibrational CD (top) spectra (solid and broken lines for the $R$ and $S$ enantiomers, respectively), and calculated spectra (bars) in the 900 and $1600 \mathrm{~cm}^{-1}$ region. For the vibrational $C D$ spectra, calculation was for the $\mathrm{R}$ enantiomer. In the experimental and calculated spectra, the peaks and troughs with the same numbers correspond to each other. The calculated vibrational CD peaks 6 and 7 are very intense with opposite signs but these peaks appear as weak peaks in the experimental spectrum, probably due primarily to the cancellation effect resulting from the small energy separation 
electronic absorption bands and redox couples, since gradual changes in properties are generally observed by the change of the ionic size of the central metal ion.

Other important areas of phthalocyanine synthesis include soluble polymeric Pcs [52], low temperature Pc synthesis [53], microwave Pc synthesis [54], Pc synthesis using hexadecachloro- [55] and hexadecafluorophthalocyanines [56], hexadecasubstituted Pcs [57], alkylperfluorosubstituted Pcs [58], macroporous Pc polymers [59] and Pc syntheses using hexamethyldisilazane [60] or cerium (III) [61].

Normally, phthalocyanines are simply flat twodimensional molecules and it is true that conceptually new types of Pcs are getting harder to prepare. However, there are still many properties to be unraveled, if new types of Pcs are produced. Phthalocyanines have long been described in highly esteemed scientific journals. Thus, phthalocyanines have great versatility and are of great interest to many scientists and industrialists. We are hoping that more people become interested in the chemistry of Pcs. They are the compounds of the 21 st century!

\section{Acknowledgements}

This work was partially supported by the Ministry of Education, Culture, Sports, Science, and Technology, Japan, a Grant-in-Aid for the COE project, Giant Molecules and Complex Systems, 2004.

\section{REFERENCES}

1. Braun A. Ber. Deut. Chem. Ges. 1907; 40: 27092714.

2. Byme GT, Linstead, RP and Lowe AR. J. Chem. Soc. 1934: 1017-1022.

3. Linstead RP and Lowe AR. J. Chem. Soc. 1934: 1022-1027.

4. Dent CE, Linstead RP and Lowe AR. J. Chem. Soc. 1934: 1033-1039.

5. Kobayashi $\mathrm{N}$ and Fukuda T. Expd Mater. Future 2002; 2: 34-41.

6. Elvidge JA and Linstead RP. J. Chem. Soc. 1955: 3536-3544.

7. Barrett PA, Linstead RP and Tuey GAP. J. Chem. Soc. 1939: 1809-1820.

8. Helberger JH and Rebay A. Justus Liebigs Ann. Chem. 1937; 531: 279-287.

9. Kobayashi N. In The Porpyrin Handbook, Vol. 2, Kadish KM, Smith KM, Guilard R. (Eds.) Academic Press: New York, 1999; pp 301-360.

10. Kobayashi N. In The Porpyrin Handbook, Vol. 15, Kadish KM, Smith KM, Guilard R. (Eds.) Academic Press: New York, 2003; pp 161-262.

11. Kobayashi N. In Phthalocyanines-Properties and Applications, Vol. 2, Leznoff CC, Lever
ABP. (Eds.) VCH: New York, 1992; pp 97-161.

12. Makarova EA, Kopranenkov VN, Shevtsov VK and Luk'yanets EA. Chem. Heterocycl. Comp. (Engl. Trans.) 1989; 1159-1164.

13. Leznoff CC and McKeown NB. J. Org. Chem. 1990; 55: 2186-2190.

14. Kobayashi N, Kondo R, Nakajima $S$ and Osa $T$. J. Am. Chem. Soc. 1990; 112: 9640-9641.

15. Kobayashi N, Ashida T and Osa T. Chem. Lett. 1992; 2031-2034.

16. Rodriguez-Morgade MS, Torre G and Torres T. In The Porpyrin Handbook, Vol. 15, Kadish KM, Smith KM, Guilard R. (Eds.) Academic Press: New York, 2003; pp 125-160.

17. Kobayashi Nand KonamiH. In PhthalocyaninesProperties and Applications, Vol. 4, Leznoff CC, Lever ABP. (Eds.) VCH: New York, 1996; pp 343-404.

18. Tse UH, Goel A, Hu M, Lever ABP and Leznoff CC. Can. J. Chem. 1993; 71: 742-753.

19. Mack J, Kobayashi N, Leznoff CC and Stillman MJ. Inorg. Chem. 1997; 36: 5624-5634.

20. Kobayashi N, Ishizaki $T$, Ishii $K$ and Konami $H$ J. Am. Chem. Soc. 1999; 121: 9096-9110.

21. Claessens CG and Torres T. Chem. Rev. 2002; 102: 835-853.

22. Kobayashi $\mathbf{N}$ and Fukuda T. J. Am. Chem. Soc. 2002; 124: 8021-8034.

23. Aoudia M, Cheng G, Kennedy VO, Kenney ME and Rodgers MA. J. Am. Chem. Soc. 1997; 119: 6029-6039.

24. Kobayashi N, Mack J, Ishii $\mathrm{K}$ and Stillman MJ. Inorg. Chem. 2002; 41: 5350-5363.

25. Linssen TG and Hanack M. Chem. Ber. 1994; 127: $2051-2057$.

26. Nicolau M, Cabezon B and Torres T. Coord. Chem. Rev. 1999; 190-192: 231-243.

27. Rodriguez-Morgade MS, Cabezon B, Esperanza $S$ and Torres T. Chem. Eur. J. 2001; 7: 24072413.

28. Silbert JW, Baumann TF, Williams DJ, White AJP, Barrrett AG and Hoffman BM. J. Am. Chem. Soc. 1996; 118: 10487-10493.

29. Garrido MA, Lange SJ, Beall S, Mani NS, Williams DJ, White AJP, Barrett AGM and Hoffman BM. J. Org. Chem. 1997; 62: 92849289.

30. Leznoff CC. Can. J. Chem. 2000; 78: 167-183.

31. Makarova EA, Fukuda T, Luk'yanets EA and Kobayashi N. in preparation. (For a review see Schmid G, Sommerauer M, Geyer M and Hanack M. In Phthalocyanines-Properties and Applications, Vol. 4, Leznoff CC, Lever ABP. (Eds.) VCH: New York, 1996; 1-18.

32. Chambrier I, Cook MJ and Wood PT. Chem. Commun. 2000: 2133-2134.

33. Burnham PM, Cook MJ, Gerrard LA, Heeney 
MJ and Hughes DL. Chem. Commun. 2003: 2064-2065.

34. Kobayashi N, Fukuda T, Ueno $\mathrm{K}$ and Ogino $\mathrm{H}$. J. Am. Chem. Soc. 2001; 123: 10740-10741.

35. Fukuda T, Homma $S$ and Kobayashi N. Chem. Commun. 2003: 1574-1575.

36. Kobayashi N. Coord. Chem. Rev. 2001; 219 221: 99-123 (this review explains chiral Pcs reported until the year 2001).

37. Engelkamp H, Middlebeek S and Nolte RJM. Science. 1999; 284: 785-788.

38. Cook AS, Williams BG, White AJP, Williams DJ, Lange SJ, Barrett AGM and Hoffman BM. Angew. Chem. Int. Ed. Engl. 1997; 36: 760761.

39. Fox JM, Katz TJ, Elshocht T, Verviest T, Kauranen M, Persoons A, Thongpangchan T, Kraus T and Brus L. J. Am. Chem. Soc. 1999; 121: 3453-3459 (optically inactive helicenefused Pcs are also known: Sooksimuang $T$ and Mandal BK. J. Org. Chem. 2003; 68: 652-655).

40. Kobayashi N, Kobayashi $\mathrm{Y}$ and Osa T. $J . A m$. Chem. Soc. 1993; 115: 10994-10995.

41. Kobayashi N, Higashi $R$, Titeca BC, Lamote $F$ and Ceuleman A. J. Am. Chem. Soc. 1999; 121: 12018-12028.

42. Kobayashi $\mathbf{N}$ and Fukuda T. Chem. Lett. 2004: 32-33.

43. Terekhov DS, Nolan KJM, McArthur CR and Leznoff CC. J. Org. Chem. 1996; 61: 30343040.

44. Leznoff $\mathrm{CC}, \mathrm{Li} \mathrm{ZP}$, Isago $\mathrm{H}, \mathrm{D}^{\prime}$ Ascanio $\mathrm{AM}$ and Terekhov DS. J. Porphyrins Phthalocyanines. 1999; 3: 406-416.

45. Cook MJ and Heeney MJ. Chem. Eur. J. 2000; 6: 3958-3967.

46. Leznoff CC, Lam H, Marcuccio SM, Nevin WA, Janda P and Kobayashi N. Chem. Commun.
1986: 699-700.

47. Kobayashi N. Coord. Chem. Rev. 2002; 227: 129-152 (this review introduces dimers, trimers, and oligomers of Pcs and related compounds).

48. Torre G, Martinez-Diaz MV, Ashton PR and Torres T. J. Org. Chem. 1998; 63: 8888-8893.

49. Kobayashi $\mathrm{N}$ and Ogata $\mathrm{H}$. Eur. J. Inorg. Chem. 2004: 906-914.

50. Jiang $\mathrm{J}$, Kasuga $\mathrm{K}$ and Arnold DP. In Spramolecular Photosensitive and Electroactive Materials, Vol. 2, Nalwa HS. (Ed.) Academic Press: New York, 2001; pp 113-210.

51. Jiang J, Bian Y, Furuya F, Liu W, Choi MTM, Kobayashi N, Li HW, Yang Q, Mak TCW and Ng DKP. Chem. Eur. J. 2001; 7: 5059-5069.

52. McKeown NB. Phthalocyanine Materials: Synthesis, Structure and Function, Cambridge University Press, Cambridge, 1998.

53. Leznoff CC, D'Ascanio AM, and Yildiz, SZ. $J$. Porphyrins Phthalocyanines. 2000; 3: 406-416.

54. Shaabani A, Safari N, Bazgir, A, Bahadoran F, Sharifi N and Jamari PR. Synth. Comm. 2003; 33: $1717-1725$.

55. Gregory P. J. Porphyrins Phthalocyanines. 1999, 3, 468-476.

56. Leznoff CC and Sosa-Sanchez JL. Chem. Commun. 2004: 338-339.

57. Eberhardt W and Hanack M. Synthesis. 1997: 95-100.

58. Gorun SM, Bench BA, Carpenter G, Beggs MW, Mague JT and Ensley HE. J. Fluorine Chem. 1998; 91: 37-40.

59. Mckeown NB, Makhseed $S$ and Budd PM. Chem. Commun. 2002: 2780-2781.

60. Uchida H, Reddy PY, Nakamura S and Toru T. J. Org. Chem. 2003; 68: 8736-8738.

61. Lee C-H and Ng DKP. Tetrahedron Lett. 2002; 43: 4211-4214. 